\title{
An outbreak of nosocomial infectious bacteria on the surfaces of medical instruments and the wounds of patients shortly after admission to the newly built burn intensive care unit
}

Jia Huang ( $\sim$ jiahuang0909@163.com )

People's Hospitol of Xindu district https://orcid.org/0000-0001-6657-8479

Jie Zhu

Affiliated hospital of Jiangnan University

Lan Tang

People's hospital of Xindu district

Huali Cheng

affiliate hospital of Jiangnan University

Guozhong Lv

affiliate Hospital of Jiangnan University

Haiyan Pan

Affiliated Hospital of Jiangnan University

Chris Wakim

Brown University

Qinfang Yuan

Affiliated Hospital of Jiangnan University

Pingping Lin

Affiliated Hospital of Jiangnan University

Rongfang Chen

Affiliated Hospital of Jiangnan University

Minlie Yang

Affiliated Hospital of Jiangnan University

Fenglai Yuan

Affiliated Hospital of Jiangnan University

Donglin Jiang

Affiliated Hospital of Jiangnan University

\section{Zongning Miao}

Affiliated Hospital of Jiangnan University

Bei Wang

Affiliated Hosptial of Jiangnan University 


\section{Ling Diao}

Affiliated Hospital of Jiangnan University

Minfeng Liu

Affiliated Hospital of Jiangnan University

\section{Peng Zhao}

Affiliated Hospital of Jiangnan University

\section{Feng Zhang}

Affiliated Hospital of Jiangnan University

\section{Research article}

Keywords: nosocomial infection, burn, RODAC, ERIC-PCR, relocation

Posted Date: December 9th, 2019

DOI: https://doi.org/10.21203/rs.2.18497/v1

License: (c) (i) This work is licensed under a Creative Commons Attribution 4.0 International License.

Read Full License 


\section{Abstract}

Background \& Aims: Nosocomial infection $(\mathrm{NI})$ is a significant problem in burn intensive care units (BICUs). Although a number of strict infection control practices had been carried out for preventing NI bacterium infection, the spreads or even outbreaks of these pathogens were often reported. Here we monitored the infection in a newly built BICU and tried to discover the origin and transmission route of $\mathrm{NI}$.

Methods: Five burn patients were gradually transferred or admitted into a newly built BICU. The surfaces of medical instruments were cleaned with detergent and later with chlorine disinfectant . NI bacteria colonies on surfaces of medical instruments, gloves, and burn wounds were sampled using Replicate Organism Detection and Counting (RODAC) plates. Gram-positive coccus and Gram-negative bacilli were identified, followed by antimicrobial susceptibility tests for Staphylococcus aureus and Acinetobacter baumannii. The shift of these two bacteria was depicted with a heat-map. The phylogenetic grouping was analysed based on the Enterobacterial repetitive intergenic consensus-polymerase chain reaction (ERIC-PCR) fingerprint.

Results: Colonies of S.aureus and A. baumannii were gradually discovered on several instruments during the first 5 days when the detergent was used. Conversely, since the sterilization, almost no S.aureus or A. baumannii colonies could be found. Furthermore, colonies were found on the nursing assistant's gloves at day 0 , which was attributed to her repeated gloves use after her touch of the body of the first admitted patient. Simultaneously, the wounds of the other four admitted patients were colonized with same strains of A. baumannii starting at day 1 or day 2 .

Conclusions: The $\mathrm{NI}$ bacteria rapidly colonized the surfaces of the medical instruments, which was facilitated by using detergent but was eliminated shortly after by using a disinfectant. The colonization of $\mathrm{NI}$ bacteria on the wounds of burn patients might have come from the nursing assistant's gloves.

\section{Background}

A nosocomial infection ( $\mathrm{NI})$ is a hospital-acquired/associate infection (HAl) which causes a sever threat to human health and to the lives of hospitalized patients. Nls caused by bacteria, fungi and viruses were symptomed by discharge from the wound, fever, cough, shortness breath, burning with urination or difficult urinating, headache, nausea, vomiting and diarrhea. These infections were related to person to person contact, like unclean hands, the contact of person to medical instruments such as catheters, respiratory machines, and the abuse use of antibiotics. Main types of Nls were bloodstream infection, urinary tract infection, surgical site infection, and ventilator associated pneumonia (VAP)[1]. The infection will induce the exaggerated immune response from the host characterized by an increased cytokines release, with multiple organs disfunction, like sepsis[2]. Nowadays, the preventive actions have been considered an effective way to control NI. Its inspections, including the determination and interference of the dissemination of the pathogen, have been reported as effective measures of reducing infection rates[1]. Protective isolations, such as hand washing, the use of gloves and non-permeable aprons, and 
isolation of infected patients are effective methods to prevent pathogen transmission among patients. Although lots of strict infection control practices had been carried out in order to prevent various NI bacterium, the spreads or even outbreaks of these pathogens in the hospital environment were often reported[3-5].

$51 \%$ patients have been reported by Dasgupta, et al. to have NI in ICUs. The most frequently reported sites of acquired infections in ICUs were lungs (64\%), abdominal (19\%) and blood stream (15\%) which added an additional cost of 3.5 billions USD per year [6].

In burn units, $\mathrm{NI}$ has also been a significant problem for decades. It has increased the duration of hospital stays by 18 days on average and has increased the cost of treatment by a mean of 502 US dollars in a burn unit in Turkey[7]. BICUs admit patients with moderate-to-severe burns, those in shock during the early stage of burn injuries, with aggressive infections that need to be isolated, and patients with severe inhalation injuries who need to receive tracheotomy or even a ventilator. The age, length of hospital stay, and total burn surface area of the patients with $\mathrm{NI}$ infection were higher than those non-infected patients in BICUs[8]. Although several care and isolation practices lead to the decrease of infection rates, no "gold standard" of protective strategies can be successfully used in every settings, which implied some other putative neglected factors might play important roles in the occurrence of NIs in BICUs[9].

For high-risk patients, relocation to a vacant ward is regarded as an effective measure to prevent the outbreak of a NI[10,11]. This finding may be due to fewer $\mathrm{NI}$ bacteria in the new environment in the hospital. If patients carrying $\mathrm{NI}$ bacteria were transferred into another department, an outbreak of $\mathrm{NI}$ might emerge. It has been observed that patients relocated within hospitals were responsible for spreading multidrug-resistant Klebsiella pneumoniae[12]. However, the origin and transmission route of this bacterium in a new setting in a hospital has not been fully understood. The aim of the study is to trace the transmission path of the $\mathrm{NI}$ bacteria in the new environment in the hospital and to determine an effective way to interrupt the transmission of NI bacteria to preserve a relatively sterile environment for patients in the BICU.

\section{Methods}

\section{Nosocomial infections}

The present study was conducted between the dates of $20^{\text {th }}$ September and $12^{\text {th }}$ October 2018 at the BICU of the Affiliated Hospital of Jiangnan University (Wuxi Third People's Hospital),Wuxi, China. Accompanied with the completion of the new surgical building, one hospitalized patient (BI001) was transferred from the old department to the new one at 8:20 am at day 1. From 10:15 am to 10:50 am, four other new burn patients (BI002, BI004, BI005 and BI006) were admitted into the ward.

2. Daily cleaning or sterilization for surfaces of instruments 
A nursing assistant wiped the surfaces of instruments with detergent between 9 am and 10 am and between $2 \mathrm{pm}$ and $3 \mathrm{pm}$. The surfaces included the central monitoring system (CMS), the food conveyer, the desktop of the nurse's workstation, the doctor's workstation, the dressing room, the disposal room, the treatment room, and the walls beside every sickbed. The surfaces were wiped with detergent on the first five days after these patients were transferred into the new department with detergent. From day 6 to day 22 , the nursing assistant cleaned these surfaces twice a day with chlorine disinfectant.

\section{Sample collection}

To observe the spread of S.aureus and A. baumannii on the surfaces of different instruments in the newly built BICU, before (Day 0) and after (Day 1 to Day 22) the first five patients were transferred or admitted into the new department, the bacteria cultures on the equipment surfaces were sampled. To elucidate whether the detergent or disinfectant could effectively remove $A$. baumannii on the surfaces, before (between 8 am and $9 \mathrm{am}$ ) and after (between $11 \mathrm{am}$ and $12 \mathrm{am}$ ) the detergent (from Day 3 to Day 5) or disinfectant (from Day 6 to Day 8) was used, the cultures of $A$. baumannii were sampled. To trace the origin and transmission route of $A$. baumannii, the colonies on the wound surfaces of the patients were also sampled for the first 8 days after the relocation. Cultures were sampled using RODAC plates (Gongdong, China) containing nutrient agar. The inner diameter of the plate was $5.5 \mathrm{~cm}$, and the effective sampling surface area was $24 \mathrm{~cm}^{2}$. The plates were contacted and then pressed against a flat surface for 1 minute, and then incubated at $37^{\circ} \mathrm{C}$ for $24 \mathrm{~h}$. For the computer mouse of the CMS, the plates were contacted along its curved surface.

4. Bacteria identification and antimicrobial susceptibility tests

All isolates on the RODAC plates were Gram stained. The identification of Gram-positive coccus and Gram-negative bacilli were carried out by applying Vitek 2 Compact 30 (Biomerieux, France) followed by the examination of the antimicrobial susceptibility for S. aureus and A. baumannii. The standard reference strains were ATCC25922, ATCC27853, ATCC35212, and ATCC29212.

\section{Colony observing and counting}

Any colonies on the plates were observed, counted, and expressed as colony-forming units (cfu)/100 $\mathrm{cm}^{2}$ materials. Those contamination levels that were 'too numerous to count' were described as 416.7 $\mathrm{cfu} / 100 \mathrm{~cm}^{2}$ (100 cfu/plate).

\section{Heat map of S.aureus and baumannii colony variation}

Accompanied with the identification of S.aureus and A. baumannii, their colonies on all the RODAC plates were counted. The heat map of the colony shift during the early stage (day 0 to day 22) of the department relocation was drawn according to their colony counts.

7. Molecular typing 
ERIC-PCR is often performed as a quicker and more accurate fingerprint analysis on bacterial genomes and valuable for rapid identification and homology analysis. Each tested strain has a unique fingerprint based on the amount and molecular weight of its stripes. ERIC-PCR can distinguish among the very similar strains in the same genus. The primers for ERIC-PCR were ERIC1: 5'-

ATGTAAGCTCCTGGGGATTCAC-3' and ERIC2: 5'-AAGTAAGTGACTGGGGTGAGCG-3'. Reactions were as follows: the overall reaction volume was $50 \mu \mathrm{L}$, with $2 \mu \mathrm{L}$ ERIC1 $(10 \mu \mathrm{mol} / \mathrm{L}), 2 \mu \mathrm{L}$ ERIC2 $(10 \mu \mathrm{mol} / \mathrm{L}), 5$ $\mu \mathrm{L}$ DNA template, $16 \mu \mathrm{L}$ PCR ultrapure water and (2x) $25 \mu \mathrm{L}$ Green PCR Master Mix.

\section{Cluster analysis}

DNA fingerprint fragments were given a score of ' 1 ' (presence of observed bands) or a score of ' 0 ' (absence of observed bands). Dendrogram analysis was performed using the software package NTsys-pc (Applied biostatistics Inc. Port Jefferson, NY) based on the score's matrix, with a 1\% tolerance limit and Dice's similarity coefficient using unweighted pair-group arithmetic average clustering (UPGMA). Clusters were identified according to an $80 \%$ similarity cut-off.

\section{Results}

\section{Disinfectant rather than detergent prevented the transmission of $\mathrm{NI}$ bacteria in the environment after the relocation to the newly built BICU.}

During the first 22 days after the relocation to the newly built BICU, the surfaces of the instruments in the new department were sampled using RODAC plates. All the colonies of Gram-positive coccus and Gramnegative bacilli on the plates were counted and identified. Twenty-three Gram-positive coccus or Gramnegative bacilli species were detected on the surfaces of the instruments in the department, with most of them having few colonies, except for S.aureus and A. baumannii. Therefore, we focused on the spread of these two high-density species and their drug-resistance. Before the burn patients were transferred into the new department from the old department (day 0), neither S.aureus nor $A$. baumannii could be detected on the surfaces of instruments by utilizing the RODAC plates. From day 0 to day 5 after the first five patients were transported into the department, surfaces of the instruments were wiped with detergent twice a day. By applying the RODAC plates, colonies of S.aureus were gradually discovered on the surfaces of several items one hour after cleaning instruments, including the computer mouse of the CMS, a computer mouse on the desk of the nurse's workstation, a food conveyer, and the desktop at the nurse workstation and in the dressing room, during the first five days. Simultaneously, colonies of $A$. baumannii were found on the food conveyer, the computer mouse on the desk of the doctor workstation, the wall next to a patient's bedside, and the desktop in the disposal room, in the treatment room and at the doctor workstation. Starting from day 6 , we carried out sterilization using chlorine disinfectant instead of cleaning using detergent, which led to the disappearance of S.aureus and A. baumannii from the surfaces of the instruments in the BICU (Fig 1).

Nosocomial infection bacteria on the surfaces of the instruments were quickly removed after the sterilization 
To evaluate the clearance of the $\mathrm{NI}$ bacteria by using detergent compared with disinfectant, colonies of $A$. baumannii, which was the dominant NI bacteria, were observed before and after the cleaning and sterilization procedures were carried out (Fig 2). As a result, detergent could not reduce the colony amounts on the surfaces of the food conveyer and the desktop in the treatment room, in the dressing room, and in the disposal room from day 3 to day 5 . Conversely, almost no colonies could be observed on the RODAC plates from any of the surfaces from day 6 to day 8 after the disinfectant was utilized.

\section{The prevalence of $A$. baumannii was caused by the contamination of the nursing assistant's glove.}

Since the first burn patient was transferred from the old ward into the new ward, the shift of the amount of the isolated colonies of $A$. baumannii on the wounds that were most likely to be touched of every one of the five patients was observed during the first 5 days. On day 0 , the first patient, numbered as BI001, was colonized with $A$. baumannii on his burn wound. However, from day 1 , isolates of $A$. baumannii were gradually discovered on the wounds of the other 4 patients as well. Unfortunately, despite the use of antibiotics, $A$. baumannii could no longer be eliminated due to their multidrug-resistance. We simultaneously examined the colonies on gloves of three doctors, four nurses and the only nursing assistant in the BICU before they contacted these patients. As a result, no colony was discovered on doctors' and nurses' gloves, but colonies with a concentration of $142 \mathrm{cfu} / 100 \mathrm{~cm}^{2}$ were found on the nursing assistant's gloves on day 0 . Hence, we inquired with the nursing assistant and learned that she did not replace her gloves after she had touched the body of patient BI001. To inhibit the spread of NI bacteria, the usage of an exclusive pair of gloves before she touches every patient is imperative. However, though she replaced her gloves before she wiped the surfaces of the instruments on day 1 , she did not replace her gloves after the cleaning procedure, and she still had a bacterial concentration of $125 \mathrm{cfu} / 100$ $\mathrm{cm}^{2}$ colonies on her gloves until she began to change her gloves after she cleaned the surfaces of instruments from day 2.

We examined the drug-resistance spectrums of $A$. baumannii from the burn wounds of the patients and the gloves of the nursing assistant. Furthermore, as the gloves of the nursing assistant had been replaced since day 2 before she touched the new patients, the drug-resistance of $A$. baumannii on the surfaces in the instruments could no longer be discovered. As a result, three different spectrums were discovered (Table 1). Except for approximately $1 / 4$ of the colonies from patient BI001 on day 4 (AB001a_4d with 57 $\mathrm{cfu} / 100 \mathrm{~cm}^{2}$ and AB001_4d with $201 \mathrm{cfu} / 100 \mathrm{~cm}^{2}$ ), A. baumannii isolates carried by the other four patients were all pandrug-resistant strains.

To further reveal the transmission pathway of $A$. baumannii, all colonies collected from burn wounds of the five patients, the nursing assistant's gloves, the surfaces of a food conveyer, the desktop in the disposal room, and the wall next to a patient's bedside were characterized by phylogenetic grouping based on ERIC-PCR fingerprint. All colonies on the same objects were grouped as the same cluster, except for two strains (AB001a_4d and AB001_4d) from BI004 at day 4. Therefore, we selected one isolate on the surface of every object and two isolates on the wounds of BI004, and we grouped them using ERICPCR. Twenty-two isolates were grouped into two clusters, while the remaining 2 isolates were unclustered. 
C1 was the dominant cluster, with 20 isolates. All isolates, including AB001_at day 0, from patient BI001 from day 1 to day 4 before and after being transferred to the new ward, were clustered together. Isolates from the nursing assistant's gloves on the first two days, the surfaces of a food conveyer at day 1 and other patients from day 1 to day 4 were clustered with those from patient BI001. Isolates from the surfaces on the desktop in the disposal room (DD001_1d) and wounds of patient BI004 (AB004_1d) at day 1 were grouped into cluster 2 (C2). The individual strain of WA001_1d from the surface of the wall next to a patient's bedside in the department, and another isolate from BI004 at day 4 (AB001a_4d), isolated from $\mathrm{BI} 001$, was the sole member in $\mathrm{C} 3$ or $\mathrm{C} 4$, respectively.

\section{Discussion}

The epidemic of nosocomial infectious pandrug-resistant bacteria has frustrated doctors for a long time in the BICU. Pseudomonas aeruginosa, A. baumannii and S. aureus were the most common identified NI bacteria in many burn units and BICUs ${ }^{2,[13]}$. In the BICU of our hospital, A. baumannii and $S$. aureus were two of the most popular species from 2012 to 2016 (data not shown). To prevent the spread of the NI bacteria, the most effective methods are universal screening and surveillance targeted cultures of new admissions, the emphasis of contact precautions, isolation of those carrying NI bacteria, and utilizing a universal decolonization protocol[14].

Replicate Organism Detection and Counting (RODAC) plates were utilized in the surveillance for the contamination of bacteria and fungi on the surfaces of the instruments and appliances in the department, including linen items[15], privacy curtains[16], laminar airflows[17], ultrasound probes[18], microstructured titanium used for dental implants[19], treatment tables[20], toilet seats[21], wooden tongue blades[22], walls, and floors[23]. Furthermore, RODAC plates were also used to monitor community-acquired infections, such as contamination on leather[24], fire stations[25] and the artefacts stored in archive storage sites and libraries[26]. RODAC plates could detect the total viable count of bacteria[15, 16, 24, 25, 27], the dominant species[16, 18, 20, 21, 24, 25, 27], the biofilms[19], and the evolution of bacterial load[23]. RODAC plate sampling could simplify the sampling procedure and improve the monitoring efficiency, showing a more direct and real situation of surface microbe communities than swabbing.

Sampling with RODAC plates and phylogenetic grouping using ERIC-PCR, we studied the route of the NI bacteria transmission shortly after the relocation of the BICU. In the present study, while applying RODAC plates, we found that most isolates of $\mathrm{A}$. baumannii and $\mathrm{S}$. aureus that colonized the burn wounds of patients and surfaces of equipment in BICU were multidrug-resistant and even pandrug-resistant strains. The execution of infection control and prevention standards, including attention to hand hygiene, isolation rooms, and effective sterilization methods may reduce infections[28]. Following medical equipment cleaning, no further cases of colonization by NI bacteria, such as vancomycin-resistant Enterococcus faecium, were detected in the following year[29]. However, in the present study, despite daily cleaning of the surfaces of the instruments in the department using detergent shortly after the BICU was relocated into a new environment, stains on these surfaces could not be eliminated during the first five 
days after the relocation. Cleaning fluids were reported as being contaminated with Gram-negative bacteria[30], and physical removal could not necessarily kill them[31]. Therefore, cleaning with a detergent might be responsible for the maintenance and even increase of the microbiological loads on the surfaces of medical instruments. Conversely, almost no colonies could be detected as soon as the nursing assistant wiped the surfaces utilizing chlorine disinfectant instead of the detergent. It revealed that to cut off the possible NI bacteria transmission pathway, which was patient-environment-patient in the BICU, the surfaces of instruments must be wiped with disinfectant.

Microbial contamination could be transferred via cloths, hands or utensils[31]. NIs spreading between patients was often attributed to contaminated hands of healthcare workers and environment[30]. However, in the current study, we did not find any colonies on doctors' or nurses' gloves. Meanwhile, although the nursing assistant wore gloves in our BICU, she did not understand the importance of changing to a fresh pair of gloves before she contacted the body of the next patient. Based on the molecular typing analysis, the first patient (BI001) was discovered as being colonized by A. baumannii on his burn wound, which was in the same cluster (C1) with the strains isolated from the nursing assistant's gloves at day 1 and day 2 after the relocation. From then on, the colonies of $A$. baumannii were gradually isolated from the burn wounds of other patients. Based on the dendrogram analysis and the occurrence time of these strains, we deduced that the spread pathway of $A$. baumannii might be from patient BI001 via the nursing assistant's gloves to the other four patients shortly after the relocation. Patient BI001 harboured another strain of $A$. baumannii (AB001a_4d) on his burn wounds at day 4 according to the cluster analysis. This strain did not spread to other patients, possibly due to its relative low density, the utilization of new gloves before contacting the next patients from day 3 , and its drug-sensitivity to most of the antibiotics. This finding also implied that two strains of $A$. baumannii might implant on the wounds of patient BI001, and only the dominant and pandrug-resistant strain began to prevail in the department shortly after the relocation. Furthermore, though the nursing assistant began to use new gloves exclusively for surface cleaning of every instrument since day 3 , the outbreak of NI bacteria could still be discovered on some surfaces. The presence of NI bacteria might be attributed to the usage of detergent, as almost no colonies could be detected on the surfaces of instruments after the detergent was substituted by the disinfectant.

\section{Conclusions}

To our knowledge, RODAC plates were usually used for the detection of bacterial contamination in the environments of the administrations for the factories of foods, feeds, and drugs and only few reports can be found about ROADC plate being used for $\mathrm{NI}$ monitoring in BICU. In the current study, we suggested the monitoring using this plate could be a promising techniques in the surveillance of $\mathrm{NI}$. I would write it as "in addition, the hospital staff play a greater in the transmission of $\mathrm{NI}$ and the implementation of the different ways of controlling it. Furthermore, some of the NI spread can be due to the detergent used which could facilitate the bacterial transmission owing to its weakness in control bacteria, and the constant switching from one medical instrument to another. The repeated use of gloves was a crucial way for bacterial transmission. We suggest a more complete guidance and training should be carried out 
to guarantee the hospital staffs, especially the nursing assistants to be well educated for NI surveillance and prevention.

\section{Abbreviations}

$\mathrm{NI}$, Nosocomial infection

BICUs, Burn Intensive Care Units, BICUs)

RODAC, Replicate Organism Detection and Counting

ERIC-PCR, Enterobacterial repetitive intergenic consensus-polymerase chain reaction

HAl, Hospital-Acquired/Associate Infection

VAP, Ventilator Associated Pneumonia (VAP)

CMS, Central Monitoring System (CMS)

\section{Declarations}

\section{Acknowledgements}

Not applicable

\section{Funding}

This report is independent research funded by the Medical technology key project of Wuxi hospital management center (YGZF1106) and Preventing medicine research project of Jiangsu province (Y2012092)supported the sample collection and carrying of the assay; Medical technology general project of Wuxi hospital management center (YGZXM1531) supported the material required for the study.

\section{Availability of data and materials}

The anonymised datasets analysed during the current study and further details on gaining access to the intervention reported within this study are available from the corresponding author on reasonable request.

\section{Authors' contributions}

Jia Huang, Jie Zhu and Feng Zhang designed the study and wrote this paper. Jie Zhu and Huali Cheng collected the RODAC plate sample of NI bacteria and experiment of ERIC-PCR. Guozhong Lv, Minlie Yang, Haiyan Pan and Qinfang Yuan focused on the sampling of the bacteria in the BICU. Chris Wakim worked 
on polishing the language. Pingping Lin and Rongfang Chen helped to perform the isolation, identification and antimicrobial susceptibility tests of the NI bacteria. Lan Tang, Minfeng Liu, Fenglai Yuan, Donglin Jiang, Zongning Miao and Bei Wang collaborated on the acquisition of data, statistics analysis and diagram painting.

*Jia Huang and Jie Zhu have equal contribution to the paper.

\section{Ethics approval and consent to participate}

The study was approved by the Ethics Committees of Wuxi No.3 people's hospital (IEC201607002). All participants were consented to participate.

\section{Consent for publication}

All participants have provided written consent to participate in this study that included for the publication.

\section{Competing interests}

The authors declare that they have no conflict of interest.

\section{References}

1.Li Y, Gong Z, Lu Y, Hu G, Cai R, Chen Z: Impact of nosocomial infections surveillance on nosocomial infection rates: A systematic review. International journal of surgery 2017, 42:164-169.

2. Hugonnet S, Sax H, Eggimann P, Chevrolet JC, Pittet D: Nosocomial bloodstream infection and clinical sepsis. Emerging infectious diseases 2004, 10(1):76-81.

3.Liu J, Yu J, Chen F, Yu J, Simner P, Tamma P, Liu Y, Shen L: Emergence and establishment of KPC-2producing ST11 Klebsiella pneumoniae in a general hospital in Shanghai, China. European journal of clinical microbiology \& infectious diseases: official publication of the European Society of Clinical Microbiology 2018, 37(2):293-299.

4.Schwaber MJ, Lev B, Israeli A, Solter E, Smollan G, Rubinovitch B, Shalit I, Carmeli Y, Israel CarbapenemResistant Enterobacteriaceae Working G: Containment of a country-wide outbreak of carbapenemresistant Klebsiella pneumoniae in Israeli hospitals via a nationally implemented intervention. Clinical infectious diseases: an official publication of the Infectious Diseases Society of America 2011, 52(7):848-855.

5. Hussein K, Rabino G, Eluk O, Warman S, Reisner S, Geffen Y, Halif L, Paul M: The association between infection control interventions and carbapenem-resistant Enterobacteriaceae incidence in an endemic 
hospital. The Journal of hospital infection 2017, 97(3):218-225.

6.Dasgupta S, Das S, Chawan NS, Hazra A: Nosocomial infections in the intensive care unit: Incidence, risk factors, outcome and associated pathogens in a public tertiary teaching hospital of Eastern India. Indian journal of critical care medicine: peer-reviewed, official publication of Indian Society of Critical Care Medicine 2015, 19(1):14-20.

7.Oncul O, Yuksel F, Altunay H, Acikel C, Celikoz B, Cavuslu S. The evaluation of nosocomial infection during 1-year-period in the burn unit of a training hospital in Istanbul, Turkey. Burns: journal of the International Society for Burn Injuries 2002, 28(8):738-744.

8. Oncul O, Oksuz S, Acar A, Ulkur E, Turhan V, Uygur F, Ulcay A, Erdem H, Ozyurt M, Gorenek L: Nosocomial infection characteristics in a burn intensive care unit: analysis of an eleven-year active surveillance. Burns: journal of the International Society for Burn Injuries 2014, 40(5):835-841.

9. Raes K, Blot K, Vogelaers D, Labeau S, Blot S: Protective isolation precautions for the prevention of nosocomial colonisation and infection in burn patients: A systematic review and meta-analysis. Intensive \& critical care nursing 2017, 42:22-29.

10.Chang CC, Cheng AC, Devitt B, Hughes AJ, Campbell P, Styles K, Low J, Athan E: Successful control of an outbreak of invasive aspergillosis in a regional haematology unit during hospital construction works. The Journal of hospital infection 2008, 69(1):33-38.

11.Gogou V, Meletis G, Tsitouras D: Control of a Multi-Drug-Resistant Acinetobacter baumannii Outbreak after Orthopedics Department Relocation. Microorganisms 2013, 1(1):158-161.

12.Richter SN, Frasson I, Franchin E, Bergo C, Lavezzo E, Barzon L, Cavallaro A, Palu G: KPC-mediated resistance in Klebsiella pneumoniae in two hospitals in Padua, Italy, June 2009-December 2011: massive spreading of a KPC-3-encoding plasmid and involvement of non-intensive care units. Gut pathogens 2012, 4(1):7.

13.Girerd-Genessay I, Benet T, Vanhems P: Multidrug-Resistant Bacterial Outbreaks in Burn Units: A Synthesis of the Literature According to the ORION Statement. Journal of burn care \& research: official publication of the American Burn Association 2016, 37(3):172-180.

14.Huang SS, Septimus E, Kleinman K, Moody J, Hickok J, Avery TR, Lankiewicz J, Gombosev A, Terpstra $L$, Hartford F et al: Targeted versus universal decolonization to prevent ICU infection. The New England journal of medicine 2013, 368(24):2255-2265.

15.Cheng VCC, Chen JHK, Wong SCY, Leung SSM, So SYC, Lung DC, Lee WM, Trendell-Smith NJ, Chan WM, Ng D et al: Hospital Outbreak of Pulmonary and Cutaneous Zygomycosis due to Contaminated Linen Items From Substandard Laundry. Clinical infectious diseases: an official publication of the Infectious Diseases Society of America 2016, 62(6):714-721. 
16.Shek K, Patidar R, Kohja Z, Liu S, Gawaziuk JP, Gawthrop M, Kumar A, Logsetty S: Rate of contamination of hospital privacy curtains on a burns and plastic surgery ward: a cross-sectional study. The Journal of hospital infection 2017, 96(1):54-58.

17.Smith EB, Raphael IJ, Maltenfort MG, Honsawek S, Dolan K, Younkins EA: The effect of laminar air flow and door openings on operating room contamination. The Journal of arthroplasty 2013, 28(9):14821485 .

18.Sanz GE, Theoret J, Liao MM, Erickson C, Kendall JL: Bacterial contamination and cleanliness of emergency department ultrasound probes. Cjem 2011, 13(6):384-389.

19.Rupf S, Idlibi AN, Marrawi FA, Hannig M, Schubert A, von Mueller L, Spitzer W, Holtmann H, Lehmann A, Rueppell $A$ et al: Removing biofilms from microstructured titanium ex vivo: a novel approach using atmospheric plasma technology. PloS one 2011, 6(10):e25893.

20.Evans MW, Jr., Campbell A, Husbands C, Breshears J, Ndetan H, Rupert R: Cloth-covered chiropractic treatment tables as a source of allergens and pathogenic microbes. Journal of chiropractic medicine $2008,7(1): 34-38$.

21.Alfa MJ, Dueck C, Olson N, Degagne P, Papetti S, Wald A, Lo E, Harding G: UV-visible marker confirms that environmental persistence of Clostridium difficile spores in toilets of patients with $C$. difficileassociated diarrhea is associated with lack of compliance with cleaning protocol.e. BMC infectious diseases 2008, 8:64.

22. Weber-Gasparoni K, Goebel BM, Drake DR, Kramer KW, Warren JJ, Reeve J, Dawson DV: Factors associated with mutans streptococci among young WIC-enrolled children. Journal of public health dentistry 2012, 72(4):269-278.

23.Frabetti A, Vandini A, Balboni P, Triolo F, Mazzacane S: Experimental evaluation of the efficacy of sanitation procedures in operating rooms. American journal of infection control 2009, 37(8):658-664.

24.Skora J, Gutarowska B, Stepien L, Otlewska A, Pielech-Przybylska K: The evaluation of microbial contamination in the working environment of tanneries. Medycyna pracy 2014, 65(1):15-32.

25.Roberts MC, Soge OO, No D, Beck NK, Meschke JS: Isolation and characterization of methicillinresistant Staphylococcus aureus from fire stations in two northwest fire districts. American journal of infection control 2011, 39(5):382-389.

26.Zielinska-Jankiewicz K, Kozajda A, Piotrowska M, Szadkowska-Stanczyk I: Microbiological contamination with moulds in work environment in libraries and archive storage facilities. Annals of agricultural and environmental medicine: AAEM 2008, 15(1):71-78.

27.Daneau G, Nduwamahoro E, Fissette K, Rudelsheim P, van Soolingen D, de Jong BC, Rigouts L: Use of RODAC plates to measure containment of Mycobacterium tuberculosis in a Class IIB biosafety cabinet 
during routine operations. International journal of mycobacteriology 2016, 5(2):148-154.

28. Tekin R, Dal T, Bozkurt F, Deveci O, Palanc Y, Arslan E, Selcuk CT, Hosoglu S: Risk factors for nosocomial burn wound infection caused by multidrug resistant Acinetobacter baumannii. Journal of burn care \& research: official publication of the American Burn Association 2014, 35(1):e73-80.

29. Herrera S, Sorli L, Perez-Saez MJ, Ruiz-Garbajosa P, Barrios C, Plasencia V, Montero M, Terradas R, Crespo M, Castells $X$ et al: Characterization and rapid control of a vancomycin-resistant Enterococcus faecium (VREF) outbreak in a renal transplant unit in Spain: The environment matters. Enfermedades infecciosas y microbiologia clinica 2017, 35(1):5-11.

30.Tacconelli E, Cataldo MA, Dancer SJ, De Angelis G, Falcone M, Frank U, Kahlmeter G, Pan A, Petrosillo $N$, Rodriguez-Bano J et al: ESCMID guidelines for the management of the infection control measures to reduce transmission of multidrug-resistant Gram-negative bacteria in hospitalized patients. Clinical microbiology and infection: the official publication of the European Society of Clinical Microbiology and Infectious Diseases 2014, 20 Suppl 1:1-55.

31.Scott E, Bloomfield SF: The survival and transfer of microbial contamination via cloths, hands and utensils. The Journal of applied bacteriology 1990, 68(3):271-278.

\section{Tables}

Table 1. Antimicrobial susceptibility of strains of $A$. baumannii 


\begin{tabular}{|c|c|c|c|c|c|c|c|c|c|c|c|c|c|c|c|}
\hline \multirow[t]{2}{*}{ Strain } & \multicolumn{15}{|c|}{$\mathrm{MIC}(\mu \mathrm{g} / \mathrm{ml})$} \\
\hline & AMP & SAM & TZP & CFZ & CTT & CAZ & CRO & FEP & ATM & IMP & GEN & TOB & CIP & LVX & SXT \\
\hline AB001_0d & $\geq 32$ & $\geq 32$ & $\geq 128$ & $\geq 64$ & $\geq 64$ & $\geq 64$ & $\geq 64$ & $\geq 64$ & $\geq 64$ & $\geq 16$ & $\geq 16$ & $\geq 16$ & $\geq 4$ & $\geq 8$ & $\geq 320$ \\
\hline NAG_Od & $\geq 32$ & $\geq 32$ & $\geq 128$ & $\geq 64$ & $\geq 64$ & $\geq 64$ & $\geq 64$ & $\geq 64$ & $\geq 64$ & $\geq 16$ & $\geq 16$ & $\geq 16$ & $\geq 4$ & $\geq 8$ & $\geq 320$ \\
\hline AB001_1d & $\geq 32$ & $\geq 32$ & $\geq 128$ & $\geq 64$ & $\geq 64$ & $\geq 64$ & $\geq 64$ & $\geq 64$ & $\geq 64$ & $\geq 16$ & $\geq 16$ & $\geq 16$ & $\geq 4$ & $\geq 8$ & $\geq 320$ \\
\hline NAG_1d & $\geq 32$ & $\geq 32$ & $\geq 128$ & $\geq 64$ & $\geq 64$ & $\geq 64$ & $\geq 64$ & $\geq 64$ & $\geq 64$ & $\geq 16$ & $\geq 16$ & $\geq 16$ & $\geq 4$ & $\geq 8$ & $\geq 320$ \\
\hline FC001_1d & $\geq 32$ & $\geq 32$ & $\geq 128$ & $\geq 64$ & $\geq 64$ & $\geq 64$ & $\geq 64$ & $\geq 64$ & $\geq 64$ & $\geq 16$ & $\geq 16$ & $\geq 16$ & $\geq 4$ & $\geq 8$ & $\geq 320$ \\
\hline AB004_1d & $\geq 32$ & $\geq 32$ & $\geq 128$ & $\geq 64$ & $\geq 64$ & $\geq 64$ & $\geq 64$ & $\geq 64$ & $\geq 64$ & $\geq 16$ & $\geq 16$ & $\geq 16$ & $\geq 4$ & $\geq 8$ & $\geq 320$ \\
\hline DD001_1d & $\geq 32$ & $\geq 32$ & $\geq 128$ & $\geq 64$ & $\geq 64$ & $\geq 64$ & $\geq 64$ & $\geq 64$ & $\geq 64$ & $\geq 16$ & $\geq 16$ & $\geq 16$ & $\geq 4$ & $\geq 8$ & $\geq 320$ \\
\hline AB005_1d & $\geq 32$ & $\geq 32$ & $\geq 128$ & $\geq 64$ & $\geq 64$ & $\geq 64$ & $\geq 64$ & $\geq 64$ & $\geq 64$ & $\geq 16$ & $\geq 16$ & $\geq 16$ & $\geq 4$ & $\geq 8$ & $\geq 320$ \\
\hline WA001_1d & $\geq 32$ & $\geq 32$ & $\geq 128$ & $\geq 64$ & $\geq 64$ & $\geq 64$ & $\geq 64$ & $\geq 64$ & $\geq 64$ & $\geq 16$ & $\geq 16$ & $\geq 16$ & $\geq 4$ & 2 & $\geq 320$ \\
\hline AB001_2d & $\geq 32$ & $\geq 32$ & $\geq 128$ & $\geq 64$ & $\geq 64$ & $\geq 64$ & $\geq 64$ & $\geq 64$ & $\geq 64$ & $\geq 16$ & $\geq 16$ & $\geq 16$ & $\geq 4$ & $\geq 8$ & $\geq 320$ \\
\hline AB002_2d & $\geq 32$ & $\geq 32$ & $\geq 128$ & $\geq 64$ & $\geq 64$ & $\geq 64$ & $\geq 64$ & $\geq 64$ & $\geq 64$ & $\geq 16$ & $\geq 16$ & $\geq 16$ & $\geq 4$ & $\geq 8$ & $\geq 320$ \\
\hline AB004_2d & $\geq 32$ & $\geq 32$ & $\geq 128$ & $\geq 64$ & $\geq 64$ & $\geq 64$ & $\geq 64$ & $\geq 64$ & $\geq 64$ & $\geq 16$ & $\geq 16$ & $\geq 16$ & $\geq 4$ & $\geq 8$ & $\geq 320$ \\
\hline AB005_2d & $\geq 32$ & $\geq 32$ & $\geq 128$ & $\geq 64$ & $\geq 64$ & $\geq 64$ & $\geq 64$ & $\geq 64$ & $\geq 64$ & $\geq 16$ & $\geq 16$ & $\geq 16$ & $\geq 4$ & $\geq 8$ & $\geq 320$ \\
\hline AB001_3d & $\geq 32$ & $\geq 32$ & $\geq 128$ & $\geq 64$ & $\geq 64$ & $\geq 64$ & $\geq 64$ & $\geq 64$ & $\geq 64$ & $\geq 16$ & $\geq 16$ & $\geq 16$ & $\geq 4$ & $\geq 8$ & $\geq 320$ \\
\hline AB002_3d & $\geq 32$ & $\geq 32$ & $\geq 128$ & $\geq 64$ & $\geq 64$ & $\geq 64$ & $\geq 64$ & $\geq 64$ & $\geq 64$ & $\geq 16$ & $\geq 16$ & $\geq 16$ & $\geq 4$ & $\geq 8$ & $\geq 320$ \\
\hline AB004_3d & $\geq 32$ & $\geq 32$ & $\geq 128$ & $\geq 64$ & $\geq 64$ & $\geq 64$ & $\geq 64$ & $\geq 64$ & $\geq 64$ & $\geq 16$ & $\geq 16$ & $\geq 16$ & $\geq 4$ & $\geq 8$ & $\geq 320$ \\
\hline
\end{tabular}

Table 1. Antimicrobial susceptibility of strains of $A$. baumannii (Continued)

\begin{tabular}{|c|c|c|c|c|c|c|c|c|c|c|c|c|c|c|c|}
\hline \multirow[t]{2}{*}{ Strain } & \multicolumn{15}{|c|}{$\mathrm{MIC}(\mu \mathrm{g} / \mathrm{ml})$} \\
\hline & AMP & SAM & TZP & CFZ & CTT & CAZ & CRO & FEP & ATM & IMP & GEN & TOB & CIP & LVX & SXT \\
\hline AB005_3d & $\geq 32$ & $\geq 32$ & $\geq 128$ & $\geq 64$ & $\geq 64$ & $\geq 64$ & $\geq 64$ & $\geq 64$ & $\geq 64$ & $\geq 16$ & $\geq 16$ & $\geq 16$ & $\geq 4$ & $\geq 8$ & $\geq 320$ \\
\hline AB006_3d & $\geq 32$ & $\geq 32$ & $\geq 128$ & $\geq 64$ & $\geq 64$ & $\geq 64$ & $\geq 64$ & $\geq 64$ & $\geq 64$ & $\geq 16$ & $\geq 16$ & $\geq 16$ & $\geq 4$ & $\geq 8$ & $\geq 320$ \\
\hline AB001_4d & $\geq 32$ & $\geq 32$ & $\geq 128$ & $\geq 64$ & $\geq 64$ & $\geq 64$ & $\geq 64$ & $\geq 64$ & $\geq 64$ & $\geq 16$ & $\geq 16$ & $\geq 16$ & $\geq 4$ & $\geq 8$ & $\geq 320$ \\
\hline AB001a_4d & $\geq 32$ & $\leq 2$ & $\leq 4$ & $\geq 64$ & $\geq 64$ & 8 & 16 & 4 & $\geq 64$ & $\leq 1$ & $\leq 1$ & $\leq 1$ & $\leq 0.25$ & $\leq 0.25$ & $\leq 20$ \\
\hline AB002_4d & $\geq 32$ & $\geq 32$ & $\geq 128$ & $\geq 64$ & $\geq 64$ & $\geq 64$ & $\geq 64$ & $\geq 64$ & $\geq 64$ & $\geq 16$ & $\geq 16$ & $\geq 16$ & $\geq 4$ & $\geq 8$ & $\geq 320$ \\
\hline AB004_4d & $\geq 32$ & $\geq 32$ & $\geq 128$ & $\geq 64$ & $\geq 64$ & $\geq 64$ & $\geq 64$ & $\geq 64$ & $\geq 64$ & $\geq 16$ & $\geq 16$ & $\geq 16$ & $\geq 4$ & $\geq 8$ & $\geq 320$ \\
\hline AB005_4d & $\geq 32$ & $\geq 32$ & $\geq 128$ & $\geq 64$ & $\geq 64$ & $\geq 64$ & $\geq 64$ & $\geq 64$ & $\geq 64$ & $\geq 16$ & $\geq 16$ & $\geq 16$ & $\geq 4$ & $\geq 8$ & $\geq 320$ \\
\hline AB006_4d & $\geq 32$ & $\geq 32$ & $\geq 128$ & $\geq 64$ & $\geq 64$ & $\geq 64$ & $\geq 64$ & $\geq 64$ & $\geq 64$ & $\geq 16$ & $\geq 16$ & $\geq 16$ & $\geq 4$ & $\geq 8$ & $\geq 320$ \\
\hline
\end{tabular}

AMP: Ampicillin; SAM: Ampicillin/sulbactam; TZP: Piperacillin/Tazobactam; CFZ: Cefazolin; CTT: Cefotetan; CAZ: Ceftazidime; CRO: Ceftriaxon; FEP: Cefepime; ATM: Aztreonam; IMP: Imipenem; GEN: Gentamicin; TOB: Tobramycin; CIP: Ciprofloxacin; LVX: Levofloxacin; SXT: Trimethoprim/sulfamethoxazole. AB001_0d: a strain from the patient numbered 
BI001 at the first day after he was transferred into the new ward. NAG: nursing assistant's glove; FC001: a colony from the food conveyer; DD001: a colony from the desktop in the disposal room. MIC: minimum inhibitory concentration.

\section{Figures}

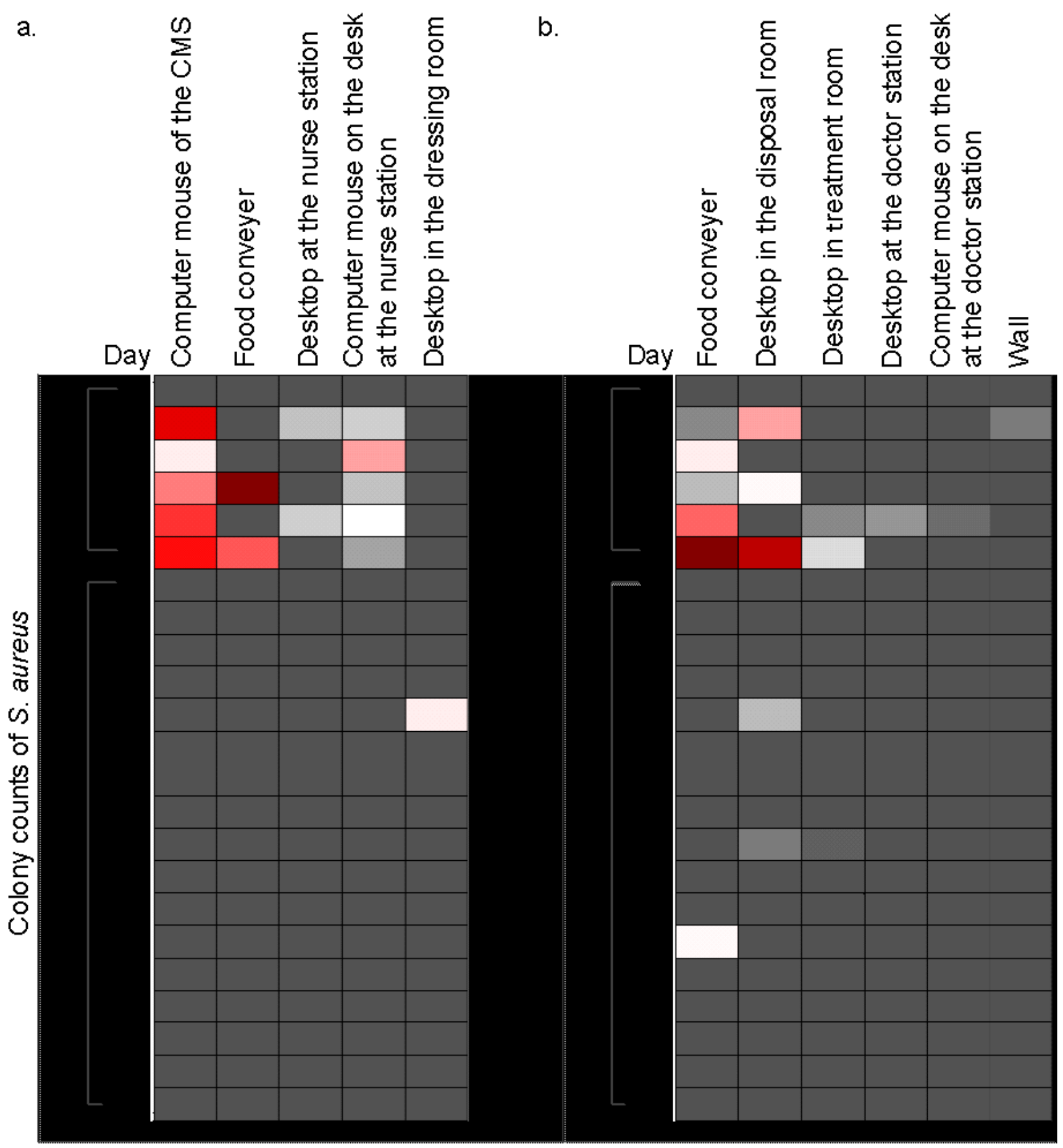

Settings in the newly built BICU

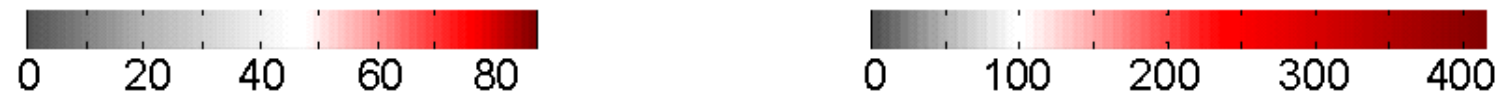

Figure 1 
Spread of S.aureus and A. baumannii on the surfaces of different instruments in the BICU was almost inhibited after the environment had been sterilized daily. The heat map shows the shift of colony counts of (a) S.aureus and (b) A. baumannii before (day 0 ) and after (day 1 to day 22) the relocation to the newly built BICU. From day 0 to day 5, detergent was used to clean the surfaces. From day 6 to day 22, disinfectant was used to sterilize the surfaces. The results are shown as cfu per unit contact area (cfu/100 cm2). CMS: Central monitoring system. Those colony counts that exceeded $416.7 \mathrm{cfu} / 100 \mathrm{~cm} 2$ (100 cfu/plate) were all presented as $416.7 \mathrm{cfu} / \mathrm{cm} 2$.

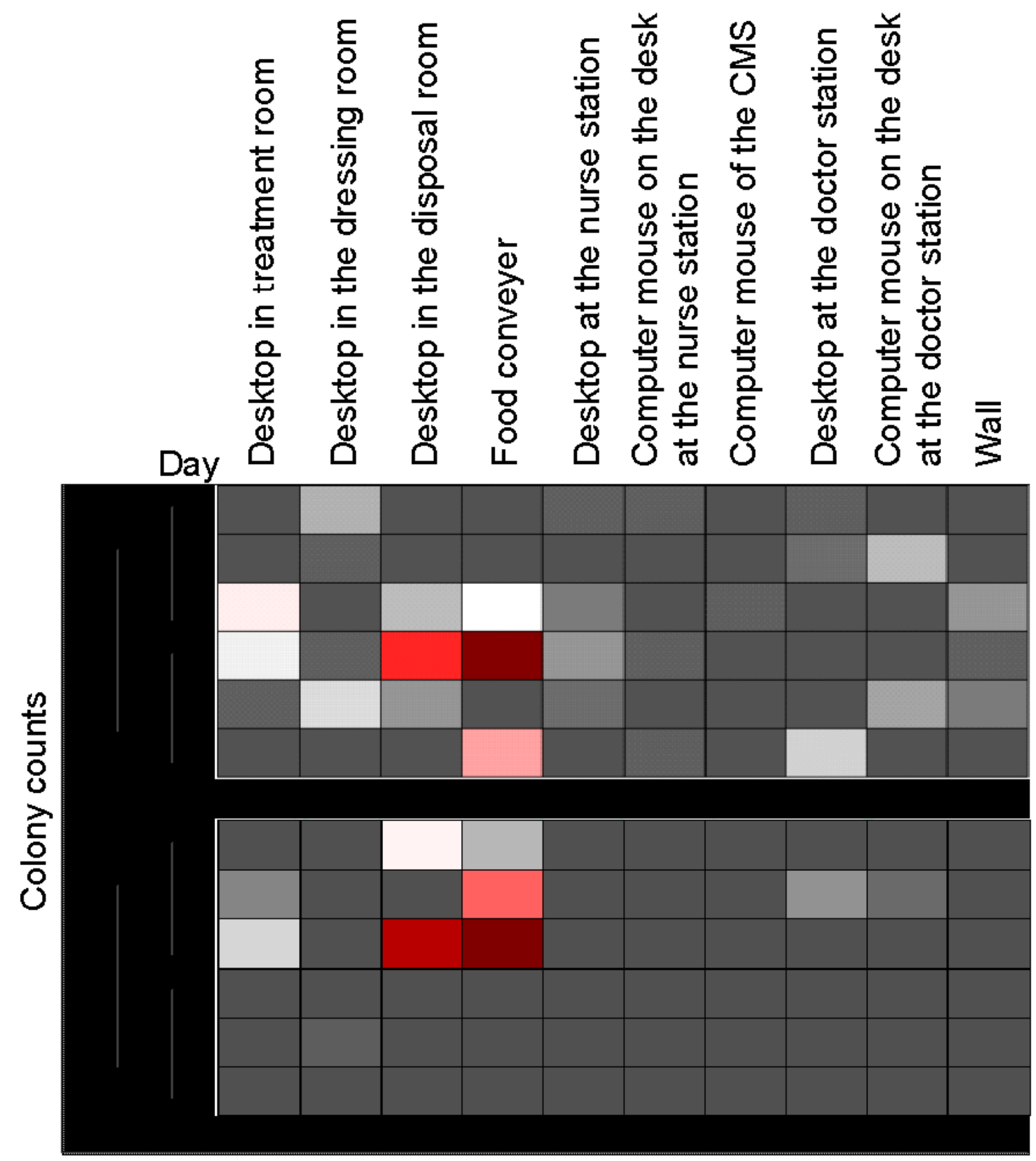

Settings in the newly built BICU

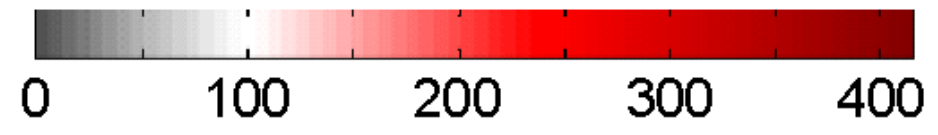


Figure 2

A. baumannii on the surfaces of different instruments were removed after sterilization. The heat map shows the colony counts (cfu/cm2) on the surfaces of 10 objects before (Pre, between 8 am and 9 am) and after (Post, between $11 \mathrm{am}$ and $12 \mathrm{am}$ ) the surfaces were cleaned using the detergent on day 3 to day 5 and sterilized using the disinfectant on day 6 to day 8 .

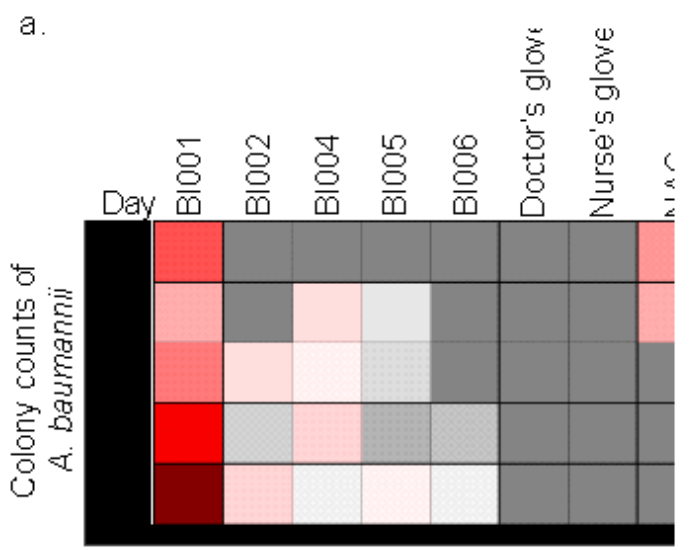

Patients

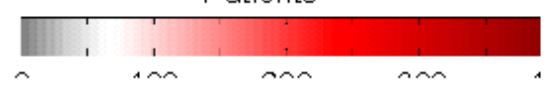

b.
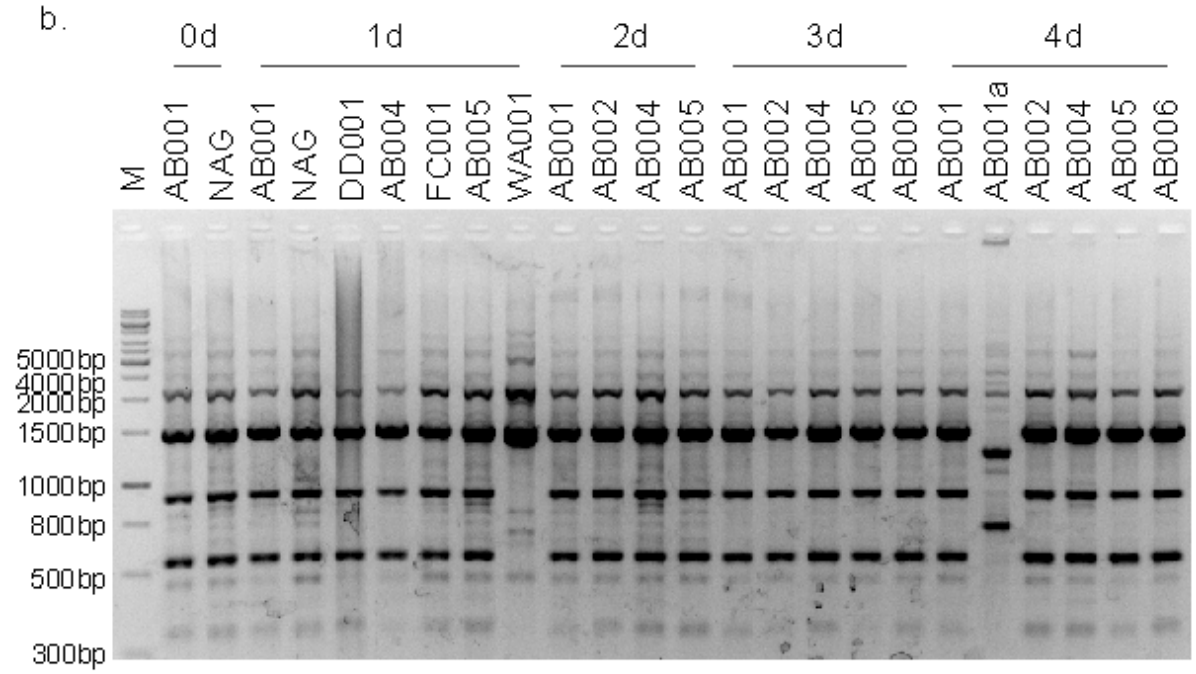

C.

Strain Phylogroup

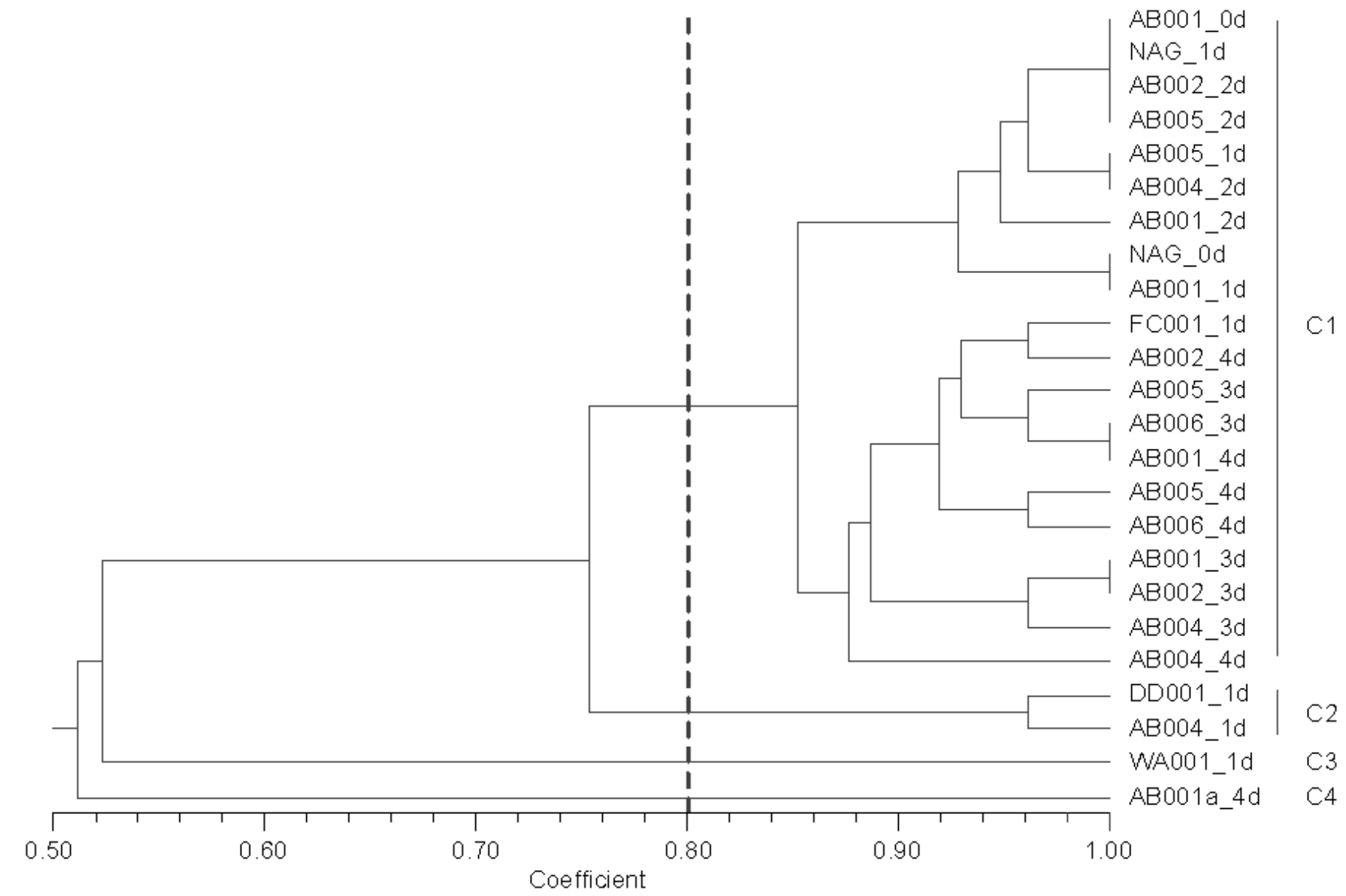


A. baumannii began to prevail on the different patients in the BICU since the A. baumannii had emerged on the nursing assistant's glove. (a) The heat map shows the colony counts (cfu/cm2) on patients numbered $\mathrm{BI001}, \mathrm{BI002}, \mathrm{BI004}, \mathrm{BI} 005$ and $\mathrm{BI} 006$ and on the doctors', nurses' and a nursing assistant's glove. (b) The electrophoresis of Eric-PCR for A. baumannii isolated from the wounds of five patients, the nursing assistant's glove and the surfaces of the food conveyer and desktop in the disposal room. (c) Cluster of 24 strains by dendrogram analysis based on the DNA fingerprint fragments. M: $1 \mathrm{~kb}$ plus marker. NAG: nursing assistant's glove. 